\title{
Thermal plasticity of a freshwater cnidarian holobiont: detection of trans-generational effects in asexually reproducing hosts and symbionts
}

\author{
Siao $\mathrm{Ye}^{1} \cdot$ Krishna N. Badhiwala $\mathbb{1}^{2} \cdot$ Jacob T. Robinson ${ }^{2,3,4} \cdot$ Won Hee Cho ${ }^{1} \cdot$ Evan Siemann ${ }^{1}$ \\ Received: 10 December 2018 / Revised: 16 February 2019 / Accepted: 15 March 2019 / Published online: 23 April 2019 \\ (c) International Society for Microbial Ecology 2019
}

\begin{abstract}
Understanding factors affecting the susceptibility of organisms to thermal stress is of enormous interest in light of our rapidly changing climate. When adaptation is limited, thermal acclimation and deacclimation abilities of organisms are critical for population persistence through a period of thermal stress. Holobionts (hosts plus associated symbionts) are key components of various ecosystems, such as coral reefs, yet the contributions of their two partners to holobiont thermal plasticity are poorly understood. Here, we tested thermal plasticity of the freshwater cnidarian Hydra viridissima (green hydra) using individual behavior and population responses. We found that algal presence initially reduced hydra thermal tolerance. Hydra with algae (symbiotic hydra) had comparable acclimation rates, deacclimation rates, and thermal tolerance after acclimation to those without algae (aposymbiotic hydra) but they had higher acclimation capacity. Acclimation of the host (hydra) and/or symbiont (algae) to elevated temperatures increased holobiont thermal tolerance and these effects persisted for multiple asexual generations. In addition, acclimated algae presence enhanced hydra fitness under prolonged sublethal thermal stress, especially when food was limited. Our study indicates while less intense but sublethal stress may favor symbiotic organisms by allowing them to acclimate, sudden large, potentially lethal fluctuations in climate stress likely favor aposymbiotic organisms. It also suggests that thermally stressed colonies of holobionts could disperse acclimated hosts and/or symbionts to other colonies, thereby reducing their vulnerability to climate change.
\end{abstract}

\section{Introduction}

Severe ecological and economic consequences of symbiotic species failure have raised concerns due to the ubiquitous distribution of symbioses in nature [1]. Consequently, we need to understand how mutualistic symbiotic species may respond to climate change in the long term [2,3]. Symbiotic species are thought to be more susceptible to environmental

Siao Ye

thomasyesiao@gmail.com

1 Department of Biosciences, Rice University, Houston, TX 77005, USA

2 Bioengineering Department, Rice University, Houston, TX 77005, USA

3 Electrical and Computer Engineering Department, Rice University, Houston, TX 77005, USA

4 Department of Neuroscience, Baylor College of Medicine, Houston, TX 77030, USA stress because the symbiosis may breakdown from malfunction in either party, plus most symbionts and some hosts reproduce asexually, where adaptation via genetic recombination is limited [4-8]. Thermal tolerance is a critical factor that determines symbiotic species stability under climate change, since many symbiotic species, from protists and plants to invertebrates, are poikilotherms that are sensitive to thermal conditions, especially temperature stress [9]. However, thermal tolerance of symbiotic species likely depends on characteristics of the host and/or symbiont.

According to the hologenome theory, holobiont (host plus symbiont) thermal tolerance is governed by both the symbiont and the host genes, yet impacts of their plasticity are overlooked [10]. Previous studies have revealed that both hosts and symbionts contribute to a holobiont's stress tolerance [11-13], and different symbiont or host strains may vary in the tolerance they confer $[14,15]$. Yet we are not aware of any published study that reports how thermal stress affects algal densities in green hydra. Our previous study on green hydra revealed that although symbiotic hydra did not bleach under heat stress as other marine 
cnidarian do, they died faster than aposymbiotic hydra, indicating being symbiotic decreased hydra thermal tolerance $[1,16,17]$. However, the long-term success of species, especially for asexually reproducing ones, under climate change is not only determined by their acute stress sensitivity but also by their plasticity [18-20]. Thermal plasticity governs the species' potential to expand thermal tolerance under prolonged stress and it consists of two main components: (1) acclimation capacity and (2) acclimation rate $[21,22]$. Acclimation capacity determines the magnitude of thermal tolerance change; acclimation rate determines how fast the organism can reach this limit [23, 24]. Both are critical to species persistence under climate change. For example, species that live close to their thermal limits with little acclimation capacity are threatened by even small temperature increases [25]; species with low absolute thermal tolerance and high acclimation capacity may persist even when the ambient temperature elevates by a large amount, but they may need to acclimate quickly or they may perish [26]. Some studies suggest that symbiont acclimation could buffer holobionts during stress [27, 28], but there is a lack of direct experimental evidence. Therefore, understanding how the host and symbiont affect holobiont acclimation is critical for predicting the impacts of long-term temperature stress on symbiotic species.

To date, how symbiont presence affects holobiont acclimation is unclear, and how acclimation in the symbiont and in the host influence holobiont thermal tolerance have not been well studied. Most experiments focused on detecting variation in acclimation responses among different holobiont populations conducted reciprocal transplants $[29,30]$. Although these studies revealed acclimation could occur in both the host and the symbiont, they did not further explore relative contributions of acclimation in each party to holobiont thermal tolerance, nor did they investigate the rate or the magnitude of such acclimation. For example, researchers found symbionts such as dinoflagellates and arbuscular mycorrhizae were able to acclimate to heat, but they did not test impacts of such acclimation on holobionts $[28,31]$. Because symbiotic organisms are often very sensitive to heat [32-34], it has been suggested that they may be less able to acclimate to thermal stress compared to aposymbiotic organisms [35]. Manipulative studies of holobiont acclimation are rarely performed and even then host and symbiont acclimation are coupled due to the difficulties in separating them [36]. In addition, past studies neglected responses of holobiont populations during thermal acclimation, instead they monitored individual physiological responses, such as transcriptome, protein content, algae density, or respiration $[21,28,30,37,38]$. The lack of holobiont population data could limit our ability to predict their population dynamics under global climate change so direct experiments and observations are needed.
Another critical process that shapes poikilotherms' success in unstable temperature environments is deacclimation, especially the rate and the extent to which the organism loses gained tolerance [39]. Cold acclimated organisms such as plants could lose their resistance during transient warm periods in winter, and are subject to damage accrued from subsequent low temperatures [40, 41]. Thus a low deacclimation rate may be beneficial so the organism could retain acquired tolerance to overcome recurring stresses. In fact, studies have revealed that such acquired plasticity could even persist in both asexually and sexually reproduced offspring, which are known as trans-generational effects [20, 42, 43]. However, for symbiotic species, such trans-generational effects have not been investigated for the host and the symbiont independently, and their impacts over multiple asexual generations is not well studied [20, 44, 45].

In this study, we conducted experiments on the freshwater cnidarian Hydra viridissima (green hydra) and derived aposymbiotic hydra (hydra without algae) to understand how thermal acclimation and deacclimation in the host (hydra) and/or the symbiont (algae) affect holobiont thermal tolerance. In addition, we tracked hydra motion activities during acclimation using microfluidic microarenas designed for hydra [46]. Hydra contract spontaneously under normal conditions, and stress could disrupt both the rhythm and magnitude of the movements [47]. Tracking hydra motions allowed us to make direct observations of the acclimation rates of symbiotic and aposymbiotic hydra, and detect algal impacts on hydra acclimation at the individual physiological level. We also reassociated acclimated hydra hosts with non-acclimated algal symbionts and vice versa, to tease apart the roles of symbiont and host in holobiont thermal acclimation and deacclimation. Although green hydra is a simple cnidarian model with low intra-host symbiont diversity in contrast to more complex symbiotic organisms such as corals, our work could have general implications for other symbiotic species [48-52].

\section{Materials and methods}

\section{Focal organisms}

Hydra viridissima is a freshwater invertebrate that hosts symbiotic green algae (Chlorella sp.) in its body [53]. The host relies on the symbiont for carbohydrates, while the symbiont relies on the host for nitrogen compounds $[54,55]$. However, aposymbiotic hydra (those that have had their symbionts experimentally eliminated) can live independently if given enough food and can be reassociated with algae [56, 57].

Hydra used in our experiment were strain 1695C acquired from Dr. Daniel Martinez at Pomona College, CA, 
and originally cultured in an $18^{\circ} \mathrm{C}$ (optimal temperature) walk-in room. For our experiments, these hydra were subjected to different thermal regimes to acquire five different acclimation states. (1) Non-acclimated (control) hydra continued to be kept at $18{ }^{\circ} \mathrm{C}$. (2) Acclimating hydra were those transferred to a $30 \pm 1{ }^{\circ} \mathrm{C}$ (sublethal heat stress) walkin room and maintained at the elevated temperature for no more than 1 month, (3) Acclimated hydra were those that had been cultured at $30^{\circ} \mathrm{C}$ for 2 months. (4) Deacclimating hydra were previously acclimated hydra transferred back to $18{ }^{\circ} \mathrm{C}$ and maintained at this optimal temperature for no more than 1 month. (5) Deacclimated hydra were previously acclimated hydra transferred back to $18{ }^{\circ} \mathrm{C}$ for 2 months. All hydra were illuminated by 3000 lux fluorescent lights with a $12: 12 \mathrm{~h}$ light-dark cycle in petri dishes in hydra medium [58].

\section{Population growth rate measurement}

We measured population growth rates of symbiotic and aposymbiotic hydra in non-acclimated, acclimating, and acclimated states. For both symbiotic and aposymbiotic hydra, eight replicates containing five non-budding hydra in the non-acclimated state were fed with brine shrimp (Brine Shrimp Direct, Inc., Ogden, UT, USA) and counted on Monday, Wednesday and Friday every week [58]. The experiment was terminated when any hydra population reached 100 polyps to exclude effects of crowding. Similarly, six replicates of non-acclimated symbiotic and aposymbiotic hydra (five polyps each) were transferred to $30^{\circ} \mathrm{C}$ respectively, then fed and counted (as described above) to acquire population growth curves of acclimating hydra. The same process was applied to acclimated hydra.

\section{Contraction activity monitoring}

We monitored symbiotic and aposymbiotic hydra contraction frequency for the first $12 \mathrm{~h}$ of acclimation. Eight symbiotic and eight aposymbiotic hydra were placed together into each of a pair of microfluidic behavioral microarenas (i.e., $N=32$ ) previously reported for constraining hydra movements in the vertical direction [46]. The microfluidic chip was fabricated using conventional microfabrication techniques and permanently bound to a glass slide. The master mold for the micro-arenas was 3D printed with $1-\mathrm{mm}$ tall parallel chambers. The two chips were transferred from the $18{ }^{\circ} \mathrm{C}$ culture room to a $24^{\circ} \mathrm{C}$ room for $2 \mathrm{~h}$ (to avoid potential heat shock effects) before being transferred to a $30^{\circ} \mathrm{C}$ water bath. We took pictures of hydra at one frame per second (EOS T3 camera, Canon USA, Melville, NY, USA). The pictures were then processed with Adobe After Effects (Adobe Systems, San Jose, CA, USA) to count the number of full contractions for each symbiotic and aposymbiotic hydra on an hourly basis. A full contraction was defined as: hydra contracts from an elongation position that is greater than $50 \%$ of the maximum length to under $20 \%$ of the maximum length. We repeated this procedure with non-acclimated hydra at $18^{\circ} \mathrm{C}$ for $6 \mathrm{~h}$ and acclimated hydra at $30^{\circ} \mathrm{C}$ for $6 \mathrm{~h}(N=32$ for each [eight symbiotic and eight aposymbiotic in two chips]).

\section{Thermal tolerance}

Non-acclimated, acclimated, and deacclimated hydra thermal tolerances were assessed by their persistence time after they were transferred from their cultivation temperature to a $38^{\circ} \mathrm{C}$ (lethal heat stress) water bath. Longer persistence time implies higher upper thermal limits. This temperature level was chosen because treatments varied at this temperature in a pilot study. The endpoint was the time hydra started to disintegrate. Twenty-four to forty non-budding polyps from each group were randomly selected for the experiment.

Acclimating symbiotic and aposymbiotic hydra thermal tolerances were assessed on day $0.5,1,2,3,5,7,14$, and 21 after they were transferred from $18{ }^{\circ} \mathrm{C}$ to $30^{\circ} \mathrm{C}$. Each time, eight to ten non-budding hydra polyps were randomly picked from each of the populations, and their persistence times were measured at $38^{\circ} \mathrm{C}$ as described above. Thermal tolerances of deacclimating hydra were assessed in a similar way after acclimated hydra were transferred back to $18^{\circ} \mathrm{C}$. During acclimation and deacclimation, hydra were fed on a weekly basis to limit budding.

To further explore how symbionts could affect hosts during long-lasting thermal stress under starvation, we kept 20 acclimated aposymbiotic hydra and 20 acclimated symbiotic hydra at $30{ }^{\circ} \mathrm{C}$ for 20 days without feeding. A control group of 20 non-acclimated aposymbiotic hydra and 20 non-acclimated symbiotic hydra were kept at $18^{\circ} \mathrm{C}$ for 20 days without feeding.

To evaluate effects of acclimation by the host vs. the symbiont on holobiont thermal tolerance and their stability across asexual generations, we reassociated hydra and algae with different thermal histories, and created four types of symbiotic hydra that had previously experienced high, elevated temperature $\left(30^{\circ} \mathrm{C}\right)$ in: (1) both the hydra and the algae, by injecting algae removed from acclimated symbiotic hydra (HA: high-temperature history algae) into acclimated aposymbiotic hydra (HH: high-temperature history hydra). (2) only the hydra, by injecting algae removed from non-acclimated symbiotic hydra (LA: low-temperature history algae) into acclimated aposymbiotic hydra. (3) only the algae, by injecting acclimated algae into non-acclimated aposymbiotic hydra (LH: low-temperature history hydra). (4) neither the hydra or the algae by injecting 


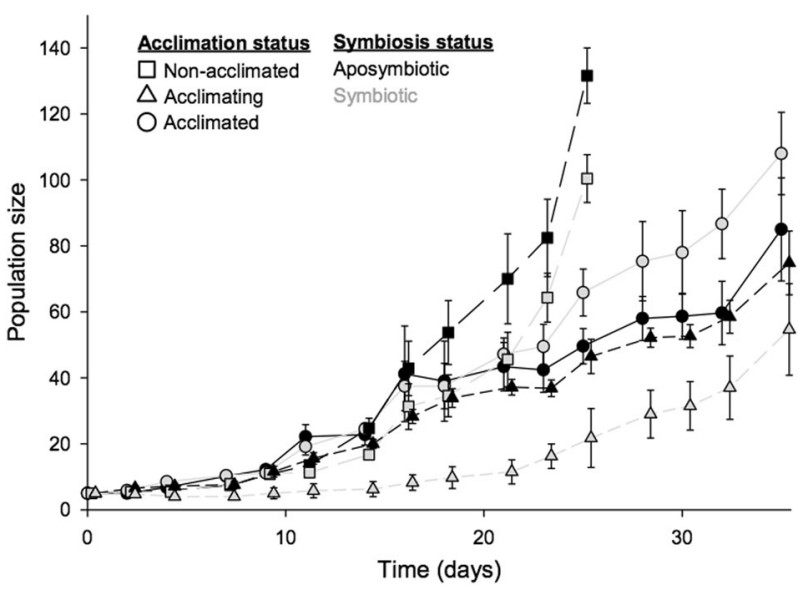

Fig. 1 Growth of symbiotic hydra (green symbols and lines) and aposymbiotic hydra (black symbols and lines) at $18{ }^{\circ} \mathrm{C}$ before acclimation (controls-squares), acclimating at $30^{\circ} \mathrm{C}$ (triangles), or acclimated at $30^{\circ} \mathrm{C}$ for 2 months (circles)

non-acclimated algae into non-acclimated aposymbiotic hydra. All hydra that turned green were picked and cultured at $18{ }^{\circ} \mathrm{C}$ until the algae density stabilized after 3 weeks, from which another three generations were derived [59-61]. We then placed 50 non-budding polyps of each type in beakers with hydra medium in a $37^{\circ} \mathrm{C}$ water bath separately. The hydra were relocated to $18^{\circ} \mathrm{C}$ after $70 \mathrm{~min}$, and their survival (determined by whether completely disintegrate or not) was tracked for 4 days.

\section{Statistical analysis}

We analyzed the dependence of population sizes (log transformed) on acclimation status (non-acclimated, acclimating, or acclimated hydra), symbiosis status and their interactions in a repeated-measures ANOVA (Proc Mixed, SAS 9.4) where time was treated as a categorical fixed variable. Then we used an ANCOVA (continuous time) and post-hoc tests to compare the exponential population growth rates for different combinations of acclimation and symbiosis statuses.

We examined the dependence of individual contraction rates on acclimation status (non-acclimated or acclimated), symbiosis status, and their interaction in an ANOVA (Proc Mixed). We used additional repeated-measures ANOVAs to analyze the dependence of individual contraction rates on symbiosis status during the acclimating and acclimated stages. We used a segmented regression [62] to analyze contraction frequencies during the first $12 \mathrm{~h}$ of acclimation (segmented package, $\mathrm{R} v 3.4 .3$ ). This enabled us to compare the breakpoints of symbiotic and aposymbiotic hydra during acclimation, which were the times they stopped exhibiting abnormal behavior.

We examined the dependence of persistence time at $38^{\circ} \mathrm{C}$ on acclimation status (acclimating or deacclimating),
Table 1 Growth rates of symbiotic and aposymbiotic hydra with different acclimation and symbiosis statuses $(\ln [\#]=a+k \times$ days $)$

\begin{tabular}{lll}
\hline Acclimation status & Symbiosis status & Growth rate $k$ \\
\hline Before acclimation (at $\left.18^{\circ} \mathrm{C}\right)$ & Symbiotic & $0.119 \pm 0.004^{\mathrm{b}}$ \\
(Non-acclimated-controls) & Aposymbiotic & $0.137 \pm 0.004^{\mathrm{a}}$ \\
During acclimation (at $\left.30^{\circ} \mathrm{C}\right)$ & Symbiotic & $0.072 \pm 0.003^{\mathrm{e}}$ \\
(Acclimating) & Aposymbiotic & $0.081 \pm 0.003^{\mathrm{d}}$ \\
After acclimation (at $\left.30^{\circ} \mathrm{C}\right)$ & Symbiotic & $0.097 \pm 0.003^{\mathrm{c}}$ \\
(Acclimated) & Aposymbiotic & $0.089 \pm 0.007^{\mathrm{cd}}$ \\
\hline
\end{tabular}

Growth rates with the same letters were not different in post-hoc tests, where "a" represents the highest growth rate and "e" represents the lowest growth rate

symbiosis status and their interaction with a repeatedmeasures ANOVA (Proc Mixed). We used an ANOVA to test the dependence of persistence time at 60 days on acclimation status (non-acclimated, acclimated, deacclimated), symbiosis status and their interaction (Proc Mixed).

We tested the dependence of starving hydra survival time under prolonged sublethal heat stress on symbiosis status using an ANOVA (Proc Glimmix, binomial distribution, logit link, SAS 9.4).

Finally, we performed a set of pairwise analyses to examine the survival time of different combinations of hydra and algal acclimation (Proc Lifetest, method= Kaplan-Meier, SAS 9.4).

\section{Results}

On a population level, acclimation status $\left(F_{2,320}=120.84\right.$, $p<0.0001)$, symbiosis status $\left(F_{1,320}=94.28, p<0.0001\right)$, and their interaction $\left(F_{2,320}=66.66, p<0.0001\right)$ had significant effects on hydra growth rate (Fig. 1). Population growth rates were highest for non-acclimated (especially aposymbiotic), intermediate for acclimated, and lowest for acclimating (higher for aposymbiotic) (Table 1).

On the individual level, hydra contraction frequency was higher after they acclimated to $30^{\circ} \mathrm{C}$ (Time $1440 \mathrm{~h}$, Fig. 2) compared with that at $18{ }^{\circ} \mathrm{C}\left(F_{1,111}=5.98, p=0.0160\right.$, Time $0 \mathrm{~h}$, Fig. 2) but the difference did not depend on symbiosis status $\left(F_{1,111}=1.13, p=0.2899\right)$. During acclimation, the contraction frequency of symbiotic and aposymbiotic hydra declined first and then recovered (Fig. 2). From hour 1 to 5, the contraction rate declined more rapidly for symbiotic than for aposymbiotic hydra $\left(F_{1,148}=14.73\right.$, $p=0.0002)$. The contraction rate was higher for aposymbiotic hydra from hours 5 to $12\left(F_{1,239}=4.92, p=0.0275\right)$ but the contraction rates did not vary with time overall $\left(F_{1,239}=3.21, p=0.0746\right)$ or between types $\left(F_{1,239}=0.31\right.$, $p=0.5813)$. The estimated breakpoint for aposymbiotic 


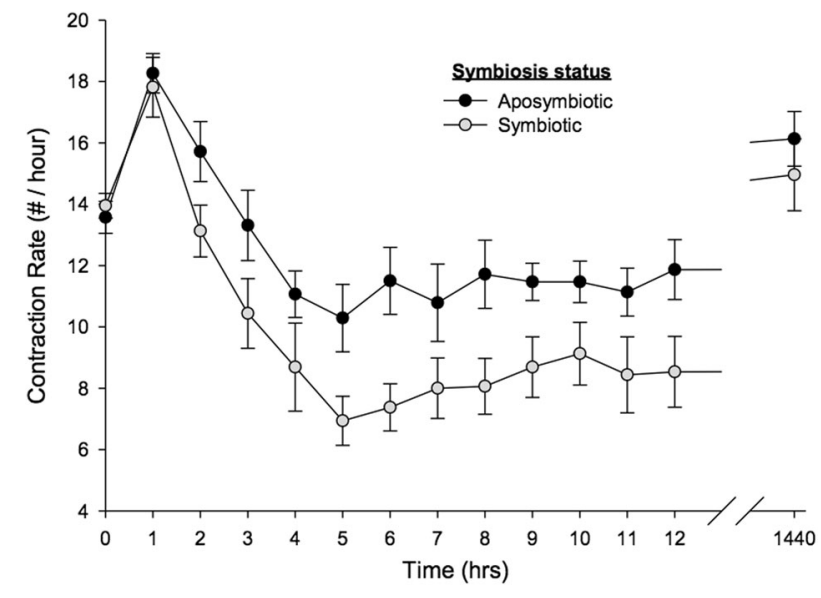

Fig. 2 Variation in contraction frequency of symbiotic and aposymbiotic hydra in non-acclimated state (time $0 \mathrm{~h}, 18^{\circ} \mathrm{C}$ ), acclimating state (time $1-12 \mathrm{~h}$, transferred to $30^{\circ} \mathrm{C}$ ), and acclimated states (time $1440 \mathrm{~h}$, $30{ }^{\circ} \mathrm{C}$ ) with time

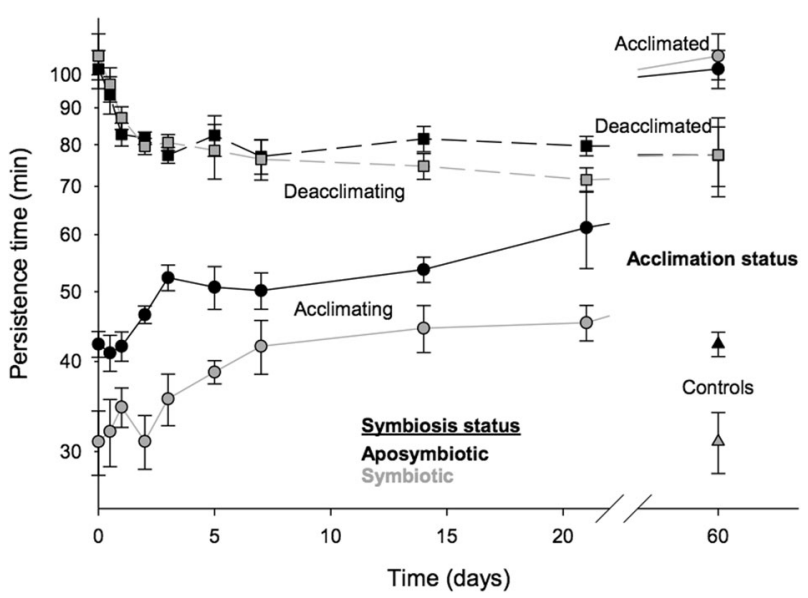

Fig. 3 Thermal tolerance of hydra heat shocked at $38^{\circ} \mathrm{C}$ in states of (1) non-acclimated controls at $18{ }^{\circ} \mathrm{C}$ (triangles). (2) acclimating at $30^{\circ} \mathrm{C}$ (circles). (3) acclimated after 2 months at $30^{\circ} \mathrm{C}$ (circles). (4) deacclimating that were transferred back to $18^{\circ} \mathrm{C}$ (squares). (5) deacclimated after 2 months back to $18{ }^{\circ} \mathrm{C}$ (squares). Controls are the starting point for acclimating hydra. Acclimated hydra are the starting point for deacclimating hydra

$(4.12 \pm 0.42 \mathrm{~h})$ and symbiotic $(4.34 \pm 0.38 \mathrm{~h})$ hydra were similar $(p=0.7060)$. Here the breakpoint refers to a switch from declining to increasing contraction frequency.

Previously acclimated hydra ("Deacclimating" in Fig. 3) had higher thermal tolerance than acclimating hydra, especially acclimating symbiotic hydra $\left(F_{1,297}=10.81, p=\right.$ 0.0011 ). Over the 21 days, persistence time at $38^{\circ} \mathrm{C}$ decreased for deacclimating hydra and increased for acclimating hydra (acclimation status effect: $F_{1,325}=56.50, p<$ 0.0001 ) but the change in persistence time did not depend on symbiosis status $\left(F_{1,325}=2.34, p=0.1267\right)$ or the interaction of acclimation and symbiosis status $\left(F_{1,326}=\right.$ 1.32, $p=0.2512$ ). After 60 days, hydra that acclimated

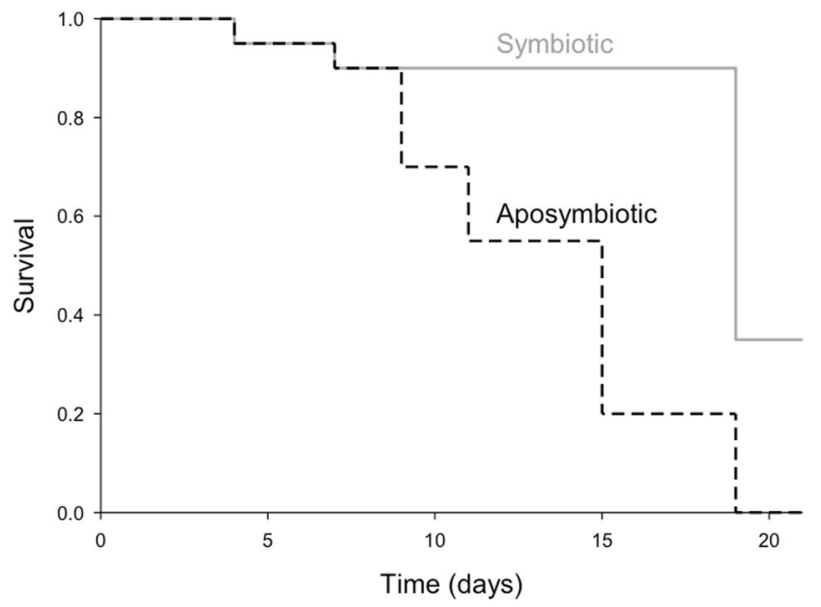

Fig. 4 Survival of symbiotic and aposymbiotic starving hydra at $30^{\circ} \mathrm{C}$ after acclimation

during this experimental period ("Acclimated" Fig. 3) had the greatest persistence time, hydra that deacclimated during this experimental period had intermediate persistence time ("Deacclimated" Fig. 3) and non-acclimated controls had the lowest persistence time $\left(F_{2,107}=80.73, p<0.0001\right)$. Symbiosis status affected persistence time only in interaction with acclimation status (main effect: $F_{1,107}=3.65, p=$ 0.0586; interaction effect: $F_{2,107}=3.22, p<0.0439$ ) with symbiotic controls having shorter persistence time than aposymbiotic controls.

Without feeding for 20 days at $30^{\circ} \mathrm{C}$, aposymbiotic hydra died more quickly than symbiotic hydra under sublethal heat stress $\left(F_{1,16}=19.31, p=0.0005\right.$, Fig. 4$)$; on the contrary, all symbiotic and aposymbiotic hydra survived a similar period at $18^{\circ} \mathrm{C}$ (data not shown).

For the crossed hydra-algae reassociation experiment, survival analysis showed that survival time varied among hydra and algae treatments (chi-sq $=77.5, p<0.0001,3 \mathrm{df}$, Fig. 5) with LH.LA (both hydra and algae were not exposed to high temperature) survival differing from the other three treatments in pairwise comparisons (all $p<0.0001$ ) but the other three treatments did not differ from each other $(0.38<p<0.96)$.

\section{Discussion}

Adaptation and acclimation, the two major mechanisms that alleviate organisms' stress in changing environments, have been proposed to be influenced by both symbionts and hosts in holobionts [10, 15, 27, 63]. Our previous studies on green hydra thermal tolerance support that holobiont stress tolerance is controlled by both the host and the symbiont, and here we looked further into impacts of their plasticity [16]. We found once symbionts are acclimated, they will not limit 


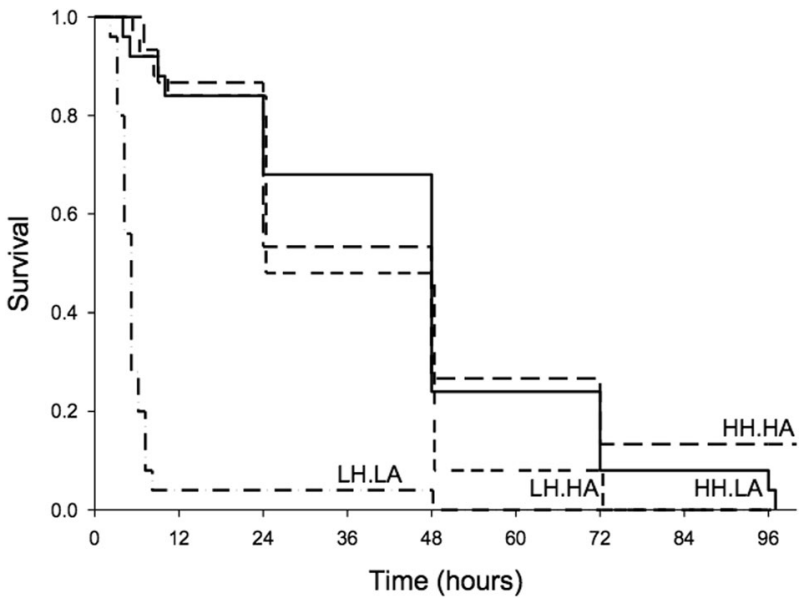

Fig. 5 Thermal tolerance of reassociated algae and hydra: (1) Both hydra and algae experienced acclimation (HH.HA). (2) Both hydra and algae were not exposed to high temperature (LH.LA). (3) Only hydra experienced acclimation (HH.LA). (4) Only algae experienced acclimation (LH.HA)

hosts' persistence under stress as previously suggested [1, 35, 64], although they could be detrimental initially during heat stress [16]; indeed, having symbionts turned out to be more advantageous during long-lasting sublethal heat stress when starvation co-occurs. We demonstrate that in addition to genetic variation in both the symbiont and the host [10], plasticity could also alter the holobiont phenotype and generate positive carryover effects over multiple asexual generations, adding another layer of complexity to hologenome theory, and imply that interactions may exist between alleles as well as epialleles of the host and the symbiont $[65,66]$.

Unlike results found in sea anemones (Exaiptasia sp.) and corals, we detected improved tolerance under heat stress at the holobiont level after acclimation [36, 38]. By measuring several distinct characteristics of symbiotic and aposymbiotic hydra, we found presence of algae had no negative impacts on hydra acclimation rate or thermal tolerance after acclimation (Fig. 2, Fig. 3). However, the more rapid decrease in contraction rate (Fig. 2) and lower persistence time of control symbiotic hydra (Fig. 3) indicate that symbiotic hydra are less thermally tolerant than aposymbiotic ones if they have no previous heat exposure, which is consistent with our past study [16]. The greater increase in persistence time of symbiotic hydra than aposymbiotic hydra gained through acclimation suggests the former has larger acclimation capacity. That the initially thermally sensitive algae became thermally tolerant after acclimation could be a result of resource re-allocation. Algae allocate more energy to carbonate production than synthesizing heat-stable saturated fatty acids under normal conditions, which benefits hosts [67-69]. Once heat stress occurs, acclimation alone could mitigate the negative impacts of these symbionts on holobiont thermal tolerance, perhaps at the cost of supplying less carbonates to hosts [70-72].

The population response results also suggest being symbiotic does not impede hydra from acclimation, and can even become beneficial when stress is not transient. While symbiotic hydra growth was greatly suppressed by elevated temperature initially, their growth rate became similar to that of aposymbiotic hydra after they were acclimated (Fig. 1). The growth of aposymbiotic hydra, on the other hand, remained relatively constant during and after acclimation. Having symbionts was more advantageous when starvation co-occurred with sublethal heat stress, as acclimated symbiotic hydra persisted for a longer time under these conditions than aposymbiotic ones (Fig. 4). This may reflect higher energy consumption by organisms at elevated temperature due to higher metabolic rates (indicated by higher contraction frequency in our experiment) [73, 74]. As the relative fitness of symbiotic and aposymbiotic hydra changed once acclimation takes place, we propose that: first, high and variable potentially lethal stress favors aposymbiotic organisms while symbiotic organisms suffer from discordant stress responses in the symbiont and the host; second, less intense stress favors symbiotic organisms as the acclimated symbiont provides supplementary functions to the host.

There is a concern that symbiotic species will be unable to withstand global climate change given high thermal sensitivity in the symbionts [35], but here we found symbionts may not limit holobiont persistence under prolonged sublethal heat stress as long as acclimation takes place. In our pilot experiments determining the appropriate acclimation temperature for hydra, we found algae but not hydra (neither symbiotic or aposymbiotic ones) were able to survive at $35^{\circ} \mathrm{C}$. Although stress responses have mainly been reported for symbionts under thermal stress, transcriptome analysis of transplanted corals showed that symbionts had significantly altered gene expression after 1 year of acclimation $[29,75,76]$. Thus a small portion of the remaining symbionts in hosts after heat stress may be able to achieve higher thermal tolerance after acclimation [33]. This is consistent with the idea that thermal sensitivity does not determine acclimation capacity [77]. Baker et al. [72] suggest symbionts may shift to parasitism in corals at elevated temperature; however, we show that short-term costs of hosting symbionts in acute environment changes appears to be offset once symbionts get acclimated. Because animals such as corals differ from hydra in within-polyp symbiont diversity, algal density stability, or transmission models, their responses to stress might not be the same as we have observed here [48, 59, 60, 78-81]. Nevertheless, we showed that symbiotic organisms could ultimately persist in the most extreme conditions of which hosts alone are capable, 
but they may need more time to reach such maxima in tolerance given lower initial tolerance.

The heat sensitivity of algae did not reappear even after 2 months of deacclimation, as we found deacclimated symbiotic and aposymbiotic hydra had similar thermal tolerances (Fig. 3). In fact, deacclimated hydra exhibited higher thermal tolerance compared with non-acclimated hydra for either symbiosis status, which further suggests the host also retained acquired thermal tolerance. The first rapidly and then steadily decreasing hydra thermal tolerance during deacclimation (without acquired tolerance being lost) supports two different modes of heat acclimation: a short-term and a long-term one [21]. The improvement in heat resistance due to previous thermal exposure could be explained by the long-term mechanism that alters baseline stress responses [82]. However, whether the symbiont will eventually return to its tolerance baseline is unclear; it is possible that over longer timescales the symbiont loses acquired tolerance so the symbiotic hydra becomes less resistant to heat again.

We discovered heat resistance was conferred to holobiont offspring by acclimated hosts and algae independently (Fig. 5), exemplifying trans-generational plasticity exists in both parties over asexual generations. Although somatic mutations could occur during asexual reproduction of both the hydra and the algae in this study, we pooled all offspring together to evaluate their thermal tolerance and did not select for those with high heat resistance. Offspring of reassociated hydra, that received either heat-exposed hosts or symbionts or both, had much greater thermal tolerance than the control group after three asexual generations at $18^{\circ} \mathrm{C}$, so acclimation rather than adaptation is likely the main contributing factor to this increase in thermal tolerance [83]. This trans-generational plasticity has also been documented in other asexual species [43, 84-86]. For example, Verhoeven and van Gurp [45] discovered parental stress history would affect offspring's stress tolerance. Dewan et al. found temperature history on parents could affect phenotypes of asexually produced offspring [84]. Thus, for asexually reproducing holobionts, acclimation in either the host or the symbiont could benefit the holobiont as well as the offspring with future stress [87]. It implies that instead of experiencing heat stress directly, symbiotic species like corals could improve their thermal tolerance by receiving either hosts and/or larvae from heat-exposed colonies [82]. Nevertheless, the relative contribution of the host and the symbiont acclimation to holobiont stress tolerance improvement might not be equal as we found in our experiment, since acclimation responses differ among holobiont species [88].

The positive trans-generational effects we observed were probably caused by epigenetic modifications rather than maternal provisioning [89]. Because the reassociated hydra had been cultured at $18{ }^{\circ} \mathrm{C}$ for three to four generations before their thermal tolerances were tested, any maternal provisioning effects should have already faded away [90]. Such positive trans-generational effects were also detected in potentially sexually reproducing corals as revealed by Putnam and Gates [87, 91, 92], although they only tested for one offspring generation, and the larvae experienced preconditioning within parents. In addition, almost exclusively asexual reproduction of hydra and algae could promote epigenetic inherence [20,53]. Since both the host and the symbiont only went through mitosis in this study, they could retain the same DNA methylation or histone modifications from their acclimated parents [20]. We suggest that maternal provisioning might influence immediate offspring fitness when the holobiont reproduces during stress, while epigenetic modification could confer tolerance for multiple asexual generations even if the holobiont is stressed before but not during reproduction. This adds another layer of complexity on the phenotype of holobionts, which appears now to be controlled by the host's and the symbiont's genomes, their genome interactions, their epigenomes, and their epigenome interactions [10, 65, 93].

\section{Conclusions}

Our study experimentally verified that acclimation could occur in both the host and the symbiont, which benefits holobionts and their offspring under stress. Although symbiotic hydra had lower thermal tolerance than aposymbiotic hydra before acclimation, they both had similar acclimation/ deacclimation rates and thermal tolerance after acclimation, indicating symbiotic hydra have greater acclimation capacity. It was unexpected to find that the presence of symbionts increased hydra fitness in prolonged sublethal heat stress when paired with starvation. To the best of our knowledge, our study is the first to show positive transgenerational effects in both hosts and symbionts of asexually reproducing holobionts, which demonstrates that symbiont presence can promote host survival rather than constrain it under climate change. It should be kept in mind that we only manipulated algal symbionts in this study, and it could be possible that associated bacterial microbiota also contribute to hydra thermal tolerance and acclimation [47, $94,95]$. Our work is based on a photosynthetic symbiosis model organism and thermal stress, yet the results may also be applicable to other kinds of abiotic stress and other symbiotic organisms. We propose symbionts provide hosts a dual insurance under climate change when the initial change is not sudden and large as symbiont acclimation can significantly improve holobiont stress tolerance. It indicates a previously stressed population not only may more readily survive subsequent stress but also could disperse acclimated 
hosts and/or symbionts to other populations and buffer them from future stress. This might guide symbiotic ecosystem management (e.g., coral reefs) by implying that a natural area experiencing less intense stress may be a priority for preservation and may even facilitate preservation of others experiencing more intense perturbations.

Acknowledgements We thank Hiroshi Shimizu and Daniel Martinez for providing materials; Daniel Wagner and Thomas Clements for assistance with micro-injections; Rudy Guerra for statistical advice; Celina Tran and Mengjia Liu for helping with the experiments; and Amy Dunham and Amanda Shore for providing advice on the manuscript.

\section{Compliance with ethical standards}

Conflict of interest The authors declare that they have no conflict of interest.

Publisher's note: Springer Nature remains neutral with regard to jurisdictional claims in published maps and institutional affiliations.

\section{References}

1. Hoegh-Guldberg O. Climate change, coral bleaching and the future of the world's coral reefs. Mar Freshw Res. 1999;50:83966.

2. Saffo MB. Invertebrates in endosymbiotic associations. Integr Comp Biol. 1992;32:557-65.

3. Nowack ECM, Melkonian M. Endosymbiotic associations within protists. Philos Trans R Soc Lond B Biol Sci. 2010;365:699-712.

4. Wernegreen JJ. Endosymbiosis. Curr Biol. 2012;22:R555-R561.

5. Toby Kiers E, Palmer TM, Ives AR, Bruno JF, Bronstein JL. Mutualisms in a changing world: an evolutionary perspective. Ecol Lett. 2010;13:1459-74.

6. Correa AMS, Baker AC. Understanding diversity in coral-algal symbiosis: a cluster-based approach to interpreting fine-scale genetic variation in the genus Symbiodinium. Coral Reefs. 2009;28:81-93

7. Baillie BK, Belda-Baillie CA, Silvestre V, Sison M, Gomez AV, Gomez ED, et al. Genetic variation in Symbiodinium isolates from giant clams based on random-amplified-polymorphic DNA (RAPD) patterns. Mar Biol. 2000;136:829-36.

8. Faeth SH. Asexual fungal symbionts alter reproductive allocation and herbivory over time in their native perennial grass hosts. Am Nat. 2009;173:554-65.

9. Guschina IA, Harwood JL. Mechanisms of temperature adaptation in poikilotherms. FEBS Lett. 2006;580:5477-83.

10. Zilber-Rosenberg I, Rosenberg E. Role of microorganisms in the evolution of animals and plants: the hologenome theory of evolution. FEMS Microbiol Rev. 2008;32:723-35.

11. Dunbar HE, Wilson ACC, Ferguson NR, Moran NA. Aphid thermal tolerance is governed by a point mutation in bacterial symbionts. PLoS Biol. 2007:5:e96.

12. Dimond JL, Holzman BJ, Bingham BL. Thicker host tissues moderate light stress in a cnidarian endosymbiont. J Exp Biol. 2012;215:2247-54.

13. Baird AH, Bhagooli R, Ralph PJ, Takahashi S. Coral bleaching: the role of the host. Trends Ecol Evol. 2009;24:16-20.

14. Berkelmans R, van Oppen MJ. The role of zooxanthellae in the thermal tolerance of corals: a 'nugget of hope' for coral reefs in an era of climate change. Proc R Soc B Biol Sci. 2006;273:2305-12.
15. Fitt WK, Gates RD, Hoegh-Guldberg O, Bythell JC, Jatkar A, Grottoli AG, et al. Response of two species of Indo-Pacific corals, Porites cylindrica and Stylophora pistillata, to short-term thermal stress: The host does matter in determining the tolerance of corals to bleaching. J Exp Mar Bio Ecol. 2009;373:102-10.

16. Ye S, Bhattacharjee M, Siemann E. Thermal tolerance in green hydra: identifying the roles of algal endosymbionts and hosts in a freshwater holobiont under stress. Microb Ecol. 2019;77:537-45.

17. Matthews JL, Sproles AE, Oakley CA, Grossman AR, Weis VM, Davy SK. Menthol-induced bleaching rapidly and effectively provides experimental aposymbiotic sea anemones (Aiptasia sp.) for symbiosis investigations. J Exp Biol. 2016;219:306-10.

18. Seebacher F, White CR, Franklin CE. Physiological plasticity increases resilience of ectothermic animals to climate change. Nat Clim Chang. 2015;5:61-66.

19. Castonguay E, Angers B. The key role of epigenetics in the persistence of asexual lineages. Genet Res Int. 2012;2012:1-9.

20. Verhoeven KJF, Preite V. Epigenetic variation in asexually reproducing organisms. Evolution. 2014;68:644-55.

21. Bay RA, Palumbi SR. Rapid acclimation ability mediated by transcriptome changes in reef-building corals. Genome Biol Evol. 2015;7:1602-12.

22. Richard J, Morley SA, Deloffre J, Peck LS. Thermal acclimation capacity for four Arctic marine benthic species. J Exp Mar Bio Ecol. 2012;424-5:38-43.

23. Ballinger RE, Schrank GD. Acclimation rate and variability of the critical thermal maximum in the lizard Phrynosoma cornutum. Phys Zool. 1970;43:19-22.

24. Calosi P, Bilton DT, Spicer JI. Thermal tolerance, acclimatory capacity and vulnerability to global climate change. Biol Lett. 2008;4:99-102.

25. Deutsch CA, Tewksbury JJ, Huey RB, Sheldon KS, Ghalambor CK, Haak DC, et al. Impacts of climate warming on terrestrial ectotherms across latitude. Proc Natl Acad Sci USA 2008;105:6668-72.

26. Sandblom E, Gräns A, Axelsson M, Seth H. Temperature acclimation rate of aerobic scope and feeding metabolism in fishes: implications in a thermally extreme future. Proc R Soc B Biol Sci. 2014;281:1490. https://doi.org/10.1098/rspb.2014.1490.

27. Bellantuono AJ, Hoegh-Guldberg O, Rodriguez-Lanetty $M$. Resistance to thermal stress in corals without changes in symbiont composition. Proceedings Biol Sci. 2012;279:1100-7.

28. Takahashi S, Yoshioka-Nishimura M, Nanba D, Badger MR. Thermal acclimation of the symbiotic alga Symbiodinium spp. alleviates photobleaching under heat stress. Plant Physiol. 2013;161:477-85

29. Kenkel CD, Matz MV. Gene expression plasticity as a mechanism of coral adaptation to a variable environment. Nat Ecol Evol. 2016;1:0014.

30. Mayfield AB, Chan P-H, Putnam HM, Chen C-S, Fan T-Y. The effects of a variable temperature regime on the physiology of the reef-building coral Seriatopora hystrix: results from a laboratorybased reciprocal transplant. J Exp Biol. 2012;215:4183-95.

31. Heinemeyer A, Ineson P, Ostle N, Fitter AH. Respiration of the external mycelium in the arbuscular mycorrhizal symbiosis shows strong dependence on recent photosynthates and acclimation to temperature. New Phytol. 2006;171:159-70.

32. Brown BE. Coral bleaching: causes and consequences. Coral Reefs. 1997;16:S129-S138.

33. Fan Y, Wernegreen JJ. Can't take the heat: high temperature depletes bacterial endosymbionts of ants. Microb Ecol. 2013;66: 727-33.

34. Montllor CB, Maxmen A, Purcell AH. Facultative bacterial endosymbionts benefit pea aphids Acyrthosiphon pisum under heat stress. Ecol Entomol. 2002;27:189-95. 
35. Wernegreen JJ. Mutualism meltdown in insects: bacteria constrain thermal adaptation. Curr Opin Microbiol. 2012;15:255-62.

36. Núñez-Pons L, Bertocci I, Baghdasarian G. Symbiont dynamics during thermal acclimation using cnidarian-dinoflagellate model holobionts. Mar Environ Res. 2017;130:303-14.

37. Mayfield AB, Fan TY, Chen CS. Physiological acclimation to elevated temperature in a reef-building coral from an upwelling environment. Coral Reefs. 2013;32:909-21.

38. Gibbin EM, Krueger T, Putnam HM, Barott KL, Bodin J, Gates $\mathrm{RD}$, et al. Short-term thermal acclimation modifies the metabolic condition of the coral holobiont. Front Mar Sci. 2018;5:10.

39. Zuther E, Juszczak I, Ping Lee Y, Baier M, Hincha DK. Timedependent deacclimation after cold acclimation in Arabidopsis thaliana accessions. Sci Rep. 2015;5:12199. https://doi.org/10. 1038/srep12199.

40. Kalberer SR, Wisniewski M, Arora R. Deacclimation and reacclimation of cold-hardy plants: Current understanding and emerging concepts. Plant Sci. 2006;171:3-16.

41. Vega SE, Palta JP, Bamberg JB. Variability in the rate of cold acclimation and deacclimation among tuber-bearing Solanum (potato) species. J Am Soc Hortic Sci. 2000;125:205-11.

42. Jablonka E, Raz G. Transgenerational epigenetic inheritance: prevalence, mechanisms, and implications for the study of heredity and evolution. Q Rev Biol. 2009;84:131-76.

43. Heard E, Martienssen RA. Transgenerational epigenetic inheritance: myths and mechanisms. Cell. 2014;157:95-109.

44. Putnam HM. Resilience and acclimatization potential of reef corals under predicted climate change stressors. PhD Thesis 2012; University of Hawai'i at Manoa.

45. Verhoeven KJF, van Gurp TP. Transgenerational effects of stress exposure on offspring phenotypes in apomictic dandelion. PLoS ONE 2012;7:e38605.

46. Badhiwala KN, Gonzales DL, Vercosa DG, Avants BW, Robinson JT. Microfluidics for electrophysiology, imaging, and behavioral analysis of Hydra. Lab Chip. 2018;18:2523-39.

47. Murillo-Rincon AP, Klimovich A, Pemöller E, Taubenheim J, Mortzfeld B, Augustin R, et al. Spontaneous body contractions are modulated by the microbiome of Hydra. Sci Rep. 2017;7:15937.

48. Rahat M. Competition between Chlorellae in chimeric infections of Hydra viridis: the evolution of a stable symbiosis. J Cell Sci. 1985;77:87-92.

49. Fay SA, Weber MX, Lipps JH. The distribution of Symbiodinium diversity within individual host foraminifera. Coral Reefs. 2009;28:717-26.

50. Carlos AA, Baillie BK, Maruyama T. Diversity of dinoflagellate symbionts (zooxanthellae) in a host individual. Mar Ecol Prog Ser. 2000;195:93-100.

51. Mars Brisbin M, Mesrop LY, Grossmann MM, Mitarai S. Intrahost symbiont diversity and extended symbiont maintenance in photosymbiotic Acantharea (Clade F). Front Microbiol. 2018;9:1998.

52. Guyomar C, Legeai F, Jousselin E, Mougel C, Lemaitre C, Simon J-C. Multi-scale characterization of symbiont diversity in the pea aphid complex through metagenomic approaches. Microbiome. 2018;6:181

53. Habetha M, Anton-Erxleben F, Neumann K, Bosch TCG. The Hydra viridis / Chlorella symbiosis. Growth and sexual differentiation in polyps without symbionts. Zoology. 2003;106: $101-8$.

54. Mews LK. The green hydra symbiosis. III. The biotrophic transport of carbohydrate from alga to animal. Proc R Soc B Biol Sci. 1980;209:377-401.

55. Cook CCB. Benefit to symbiotic zoochlorellae from feeding by green hydra. Biol Bull. 1972;142:236-42.

56. Pardy RL. Preparing aposymbiotic hydra. In: Lenhoff HM, editor. Hydra: Research Methods. US: Springer; 1983. p. 393-7.
57. Kessler E, Huss VAR, Rahat M. Species-specific ability of Chlorella strains (Chlorophyceae) to form stable symbioses with Hydra viridis. Plant Syst Evol. 1988;160:241-6.

58. Lenhoff HM, Brown RD. Mass culture of hydra: an improved method and its application to other aquatic invertebrates. Lab Anim. 1970;4:139-54.

59. Dunn K. Growth of endosymbiotic algae in the green hydra Hydra Viridissima. J Cell Sci. 1987;88:571-8.

60. Bossert P, Dunn KW. Regulation of intracellular algae by various strains of the symbiotic Hydra viridissima. J Cell Sci. 1986; 85:187-95.

61. Pardy RL, Muscatine L. Recognition of symbiotic algae by Hydra Viridis. A quantitative study of the uptake of living algae by aposymbiotic H. Viridis. Biol Bull. 1973;145:565-79.

62. Muggeo V. Segmented: An $\{R\}$ package to fit regression models with broken-line relationships. R News. 2004;4:11-7.

63. Palumbi SR, Barshis DJ, Traylor-Knowles N, Bay RA. Mechanisms of reef coral resistance to future climate change. Science (80-). 2014;344:895-8.

64. Kikuchi Y, Tada A, Musolin DL, Hari N, Hosokawa T, Fujisaki $\mathrm{K}$, et al. Collapse of insect gut symbiosis under simulated climate change. MBio 2016; 7.

65. Brucker RM, Bordenstein SR. The hologenomic basis of speciation: Gut bacteria cause hybrid lethality in the genus Nasonia. Science. 2013;341:667-9.

66. Kalisz S, Purugganan MD. Epialleles via DNA methylation: consequences for plant evolution. Trends Ecol Evol. 2004;19:309-14.

67. Tchernov D, Gorbunov MY, de Vargas C, Narayan Yadav S, Milligan AJ, Häggblom M, et al. Membrane lipids of symbiotic algae are diagnostic of sensitivity to thermal bleaching in corals. Proc Natl Acad Sci USA. 2004;101:13531-5.

68. Karmakar A, Karmakar S, Mukherjee S. Properties of various plants and animals feedstocks for biodiesel production. Bioresour Technol. 2010;101:7201-10.

69. Sachs JL, Russell JE, Lii YE, Black KC, Lopez G, Patil AS. Host control over infection and proliferation of a cheater symbiont. $\mathrm{J}$ Evol Biol. 2010;23:1919-27.

70. Wernegreen JJ, Wheeler DE. Remaining flexible in old alliances: functional plasticity in constrained mutualisms. DNA Cell Biol. 2009;28:371-82.

71. Rodríguez-Troncoso AP, Carpizo-Ituarte E, Pettay DT, Warner ME, Cupul-Magaña AL. The effects of an abnormal decrease in temperature on the Eastern Pacific reef-building coral Pocillopora verrucosa. Mar Biol. 2014;161:131-9.

72. Baker DM, Freeman CJ, Wong JCY, Fogel ML, Knowlton N. Climate change promotes parasitism in a coral symbiosis. ISME J. 2018;12:921-30.

73. Brown BE, Downs CA, Dunne RP, Gibb SW. Exploring the basis of thermotolerance in the reef coral Goniastrea aspera. Mar Ecol Prog Ser. 2002;242:119-29.

74. Gillooly JF, Brown JH, West GB, Savage VM, Charnov EL. Effects of size and temperature on metabolic rate. Science. 2001;293:2248-51.

75. Smith DJ, Suggett DJ, Baker NR. Is photoinhibition of zooxanthellae photosynthesis the primary cause of thermal bleaching in corals? Glob Chang Biol. 2005;11:1-11.

76. Strychar KB, Sammarco PW. Exaptation in corals to high seawater temperatures: low concentrations of apoptotic and necrotic cells in host coral tissue under bleaching conditions. J Exp Mar Bio Ecol. 2009;369:31-42.

77. Donelson JM, Munday PL. Thermal sensitivity does not determine acclimation capacity for a tropical reef fish. J Anim Ecol. 2012;81:1126-31.

78. Baumann J, Davies SW, Aichelman HE, Castillo KD. Coral Symbiodinium community composition across the Belize 
Mesoamerican Barrier Reef System is driven by host species and environmental variability. DoiOrg. 2017;75:154179.

79. LaJeunesse T. Diversity and community structure of symbiotic dinoflagellates from Caribbean coral reefs. Mar Biol. 2002;141: 387-400.

80. Dimond J, Carrington E. Temporal variation in the symbiosis and growth of the temperate scleractinian coral Astrangia poculata. Mar Ecol Prog Ser. 2007;348:161-72.

81. Muscatine L, Lenhoff HM. Coelenterate Biology: Reviews and New Perspectives. Academic Press: New York, USA, 1974.

82. Middlebrook R, Hoegh-Guldberg O, Leggat W. The effect of thermal history on the susceptibility of reef-building corals to thermal stress. J Exp Biol. 2008;211:1050-6.

83. Donelson JM, Salinas S, Munday PL, Shama LNS. Transgenerational plasticity andclimate change experiments: where do we go from here? Glob Chang Biol. 2018;24:13-34.

84. Dewan S, De Frenne P, Vanden Broeck A, Steenackers M, Vander Mijnsbrugge K, Verheyen K. Transgenerational effects in asexually reproduced offspring of Populus. PLoS ONE 2018;13: e0208591.

85. Latzel V, Klimešová J. Transgenerational plasticity in clonal plants. Evol Ecol. 2010;24:1537-43.

86. Verhoeven KJF, Jansen JJ, van Dijk PJ, Biere A. Stress-induced DNA methylation changes and their heritability in asexual dandelions. New Phytol. 2010;185:1108-18.

87. Putnam HM, Gates RD. Preconditioning in the reef-building coral Pocillopora damicornis and the potential for trans-generational acclimatization in coral larvae under future climate change conditions. J Exp Biol. 2015;218:2365-72.

88. Putnam HM, Davidson JM, Gates RD. Ocean acidification influences host DNA methylation and phenotypic plasticity in environmentally susceptible corals. Evol Appl. 2016;9: $1165-78$.

89. Ross PM, Parker L, Byrne M. Transgenerational responses of molluscs and echinoderms to changing ocean conditions. ICES J Mar Sci. 2016;73:537-49. Oxford University Press.

90. Räsänen K, Kruuk LEB. Maternal effects and evolution at ecological time-scales. Funct Ecol. 2007;21:408-21.

91. Combosch DJ, Vollmer SV. Mixed asexual and sexual reproduction in the Indo-Pacific reef coral Pocillopora damicornis. Ecol Evol. 2013;3:3379-87.

92. Yeoh S-R, Dai C-F. The production of sexual and asexual larvae within single broods of the scleractinian coral, Pocillopora damicornis. Mar Biol. 2010;157:351-9.

93. Bordenstein SR, Theis KR. Host biology in light of the microbiome: ten principles of holobionts and hologenomes. PLoS Biol. 2015;13:e1002226.

94. Fraune S, Bosch TCG. Long-term maintenance of species-specific bacterial microbiota in the basal metazoan Hydra. Proc Natl Acad Sci USA. 2007;104:13146-51.

95. Röthig T, Costa RM, Simona F, Baumgarten S, Torres AF, Radhakrishnan A, et al. Distinct bacterial communities associated with the coral model Aiptasia in aposymbiotic and symbiotic states with Symbiodinium. Front Mar Sci. 2016;3:234. 